Accepted Submission for Philosophy of Science Association 2010

Contributed Paper — Philosophy of Physics - about 4800 words

\title{
How to Defeat Wüthrich's Abysmal Embarrassment Argument against Space-Time Structuralism
}

\author{
F.A. Muller ${ }^{1}$
}

Summary. In his 2009 PSA Recent Ph.D. Award winning contribution to the bi-annual PSA Conference at Pittsburgh in 2008, C. Wüthrich mounted an argument against structuralism about space-time in the context of the General Theory of Relativity (GTR), to the effect that structuralists cannot discern space-time points. An "abysmal embarrassment" for the structuralist, Wüthrich judged. Wüthrich's characterisation of space-time structuralism is however incorrect. We demonstrate how, on the basis of a correct characterisation of space-time structuralism, it is possible to discern space-time points in the GTR-structures under consideration. Thus Wüthrich's argument crumbles.

1. Introduction and Overview. At the end of his magisterial monograph on the modern debate about the nature of space-time between substantivalism and relationism World Enough and Space-Time (1989: 208), J. Earman hinted at a third view overcoming the difficulties of both views. Lately space-time structuralism — or synonymously ontic structural realism about space-time - has been propounded or addressed by a number of authors as this third view. ${ }^{2}$ C. Wüthrich (2010), ironically an ex-PhD student of Earman, will have nothing of it, because structuralism has an "abysmally embarrassing" consequence. This consequence arises when we consider cosmological models generated by the General Theory of Relativity (GTR), i.e. space-times that have a unique foliation into homogeneous and isotropic 3-dimensional spaces of constant curvature; the consequence of structuralism about such 'standard cosmic space-times' is that everyone of these 3-dimensional spaces contains no more than a single space-time point. This contradicts

\footnotetext{
${ }^{1}$ Faculty of Philosophy, Erasmus University Rotterdam, Burg. Oudlaan 51, H5-10, 3062 PA Rotterdam; and Department of Physics \& Astronomy, Utrecht University, Budapestlaan 6, IGG, WG-3.11, 3584 CD Utrecht, The Netherlands, Europe; f.a.muller@uu.nl .

${ }^{2}$ See references in Wüthrich (2010) for recent papers on structuralism about space-time.
} 
the mathematical fact that such a 3-dimensional space has a non-denumerable infinitude of points.

We are going to meet this challenge head-on and out of the clash emerges a formulation of space-time structuralism that arguably fulfils Earman's hope expressed about 20 years ago. At the heart of Wüthrich's challenge lies the issue how to discern space-time points in symmetric space-times, where the points arguably share all their physico-geometrical properties. Then it seems that such space-time points are genuinely indiscernible as far as the physics goes (GTR), and what discerns them from each other is some metaphysical property, haecceittas or primitive thisness, that transcends physics. They seem qualitatively the same yet quantitatively distinct. When structuralists reject such overtly metaphysical and physics-transcending properties by adhering to Leibniz's Principle of the Identity of qualitative Indiscernibles (PIdIn), they seem committed, indeed, to the conclusion that every 3-dimensional space in a standard cosmic space-time contains a single space-time point. The way we are going to meet Wüthrich's challenge is by indicating how space-time points in such spaces, and in fact in every GTR-structure, can be discerned, on the basis of only the metric tensor after all. Thus emerges a correct version of space-time structuralism, that can and will be characterised sharply in four sonorous principles.

The paper is organised as follows. Section 2 sets the general-relativistic stage. In Section 3, we present Wüthrich's incorrect version of structuralism about space-time, and in Section 4, we expound his argument against structuralism and extend this argument beyond GTR. In Section 5, we provide a correct characterisation of structuralism in four sonorous principles and sketch, in Section 6, how to discern space-time points generally on the basis of the lightcone structure of space-time. In Section 7, we briefly address the charge of vicious circularity.

2. Standard Cosmic Space-Time Structures. We characterise GTR in the canonical manner, through its structures (also called 'models'). A GTR-structure $\mathfrak{G}$ standardly is set-theoretically defined:

$$
\mathfrak{G} \equiv\langle\mathcal{M}, \mathcal{T}(\mathcal{M}), \mathcal{C}(\mathcal{M}), \mathcal{A}(\mathcal{M}), g, T\rangle
$$

where base set $\mathcal{M}$ is a 4 -dimensional Lorentzian geometric differentiable manifold ${ }^{3}$ (the set of space-time points), $\mathcal{T}(\mathcal{M})$ is a (paracompact, Hausdorff) topological subset-family of $\mathcal{M}, \mathcal{C}(\mathcal{M}) \subset \mathcal{T}(\mathcal{M})$ is an open cover of $\mathcal{M}, \mathcal{A}(\mathcal{M})$ is a maximal atlas (co-ordinate charts of the covering regions, smoothly fitted together), and $g$ is the metric tensor of $\operatorname{rank} 2$ on $\mathcal{M} .^{4}$

\footnotetext{
${ }^{3}$ For GTR, it is sufficient to consider $C^{2}$-manifolds, because solutions of Einstein's field equations need only be differentiable twice. Lorentzian geometric manifolds come by definition equipped with a metric tensor $g$ of signature +2 .

${ }^{4}$ Other geometrical objects, like the affine connexion $\Gamma$, the Christoffel symbols $\{\therefore\}$, the Ricci tensor,
} 
$\operatorname{Hom}(\mathfrak{G})$ is the group of homeomorphisms, which preserve the topological structure of $\mathfrak{G}$, encoded in $\mathcal{T}(\mathcal{M})$. $\operatorname{Diff}(\mathfrak{G})$ is the group of diffeomorphisms, which preserve the differentiability structure, encoded in the $\mathcal{C}(\mathcal{M})$ cum $\mathcal{A}(\mathcal{M})$. Sym(G) is the group of spatio-temporal symmetries, or isometries, which preserve the geometrical structure, encoded in $g$. When we extend $\operatorname{Sym}(\mathfrak{G})$ with the dilatations, we obtain the conformal group, also called the causal group, and therefore denoted by $\operatorname{Caus}(\mathcal{M})$, because it preserves 'the lightcone structure' and by implication the usual causal relations one can define in $\mathcal{M}$. $\operatorname{Aut}(\mathfrak{G})$ is the group of automorphisms, which leave the entire structure $\mathfrak{G}$ invariant. These composition-groups of one-one mappings $\mathcal{M} \rightarrow \mathcal{M}$ are related as follows:

$$
\operatorname{Aut}(\mathfrak{G}) \subseteq \operatorname{Sym}(\mathfrak{G}) \subseteq \operatorname{Caus}(\mathfrak{G}) \subseteq \operatorname{Diff}(\mathfrak{G}) \subseteq \operatorname{Hom}(\mathfrak{G}) \subset \Pi(\mathcal{M})
$$

where $\Pi(\mathcal{M})$ is the set of one-one mappings (permutations) on $\mathcal{M}$. We emphasise that $\operatorname{Sym}(\mathfrak{G})$ should not be confused with $\operatorname{Sym}(\mathrm{GTR})$, the symmetry group of the theory, which is the group of all diffeomorphisms between all GTR-structures.

This was the marble; now comes the wood: enter GTR. The final item in structure $\mathfrak{G}(1)$ is the energy-momentum tensor $T$ of rank 2 , which codifies the distribution of matter, and is required to be related to $g$ according to Einstein's field equations. Matter $(T)$ determines the geometry $(g)$ of space-time, and the geometry of space-time determines the behaviour of matter: free particles and bodies move along geodesics according to the geodesic equation ('the equation of free motion'). If the field equations with a given $T$ have no solution, there is no ensuing GTR-structure either; if there is a solution (up to isometry), we have an ensuing GTR-structure; if there are more (non-isometric) solutions, there are more ensuing GTR-structures.

Finally, when there is no energy-momentum $(T=0)$, hence no matter, $g$ is the LorentzMinkowski metric $(\eta)$, and $\mathcal{T}(\mathcal{M})$ is some Zeeman topology $(Z(\mathcal{M}))$, we obtain the fixed space-time structure of the Special Theory of Relativity (STR), known as Minkowski space-time:

$$
\mathfrak{M}=\langle\mathcal{M}, Z(\mathcal{M}), \eta\rangle .
$$

The spatial part of $\eta$ is the Euclidean metric tensor, so that all simultaneity spaces are the same flat 3-dimensional Euclidean spaces $\left(\mathbb{E}^{3}\right)$; and $\operatorname{Sym}\left(\mathbb{E}^{3}\right)$ is the familiar Euclidean group of displacements and rotations. The spatio-temporal symmetry group $\operatorname{Sym}(\mathfrak{M})$ is the Poincaré group. $\mathfrak{M}$ is also spatio-temporally homogenous, in that group $\operatorname{Sym}(\mathfrak{M})$ acts transitively on the whole of $\mathcal{M}$.

Let us now call a GTR-structure $\mathfrak{G}(1)$ a cosmic space-time structure iff there is an open interval $I \subset \mathbb{R}$ and a complete, connected 3-dimensional Riemannian differentiable

\footnotetext{
etc., are all determined by $g$ and therefore omitted from six-tuple (1) - other elements in the six-tuple usually are also omitted, such as $\mathcal{T}(\mathcal{M}), \mathcal{C}(\mathcal{M})$ and $\mathcal{A}(\mathcal{M})$, but we have put them in for the sake of future reference. Whether $\mathfrak{G}(1)$ should be taken to represent 'the geometry of space-time', or whether some far more sparser structure represents it, such as $\langle\mathcal{M}, g\rangle$, we need not decide now.
} 
manifold having base set $\Sigma$ such that $\mathcal{M}$ is isomorphic to $I \times \Sigma$, and the so-called cosmic time function $f: I \rightarrow \Sigma, t \mapsto \Sigma_{t}$ is differentiable (in an appropriate sense, see McCabe (2004)). For the sake of brevity, we call the 3-dimensional spatial submanifolds into which cosmic space-time is foliated cosmic spaces. One now speaks of 3+1-dimensional spacetime. Every cosmic space $\Sigma_{t}$ is a 'snapshot' of space-time $\mathcal{M}$ at cosmic time $t$. We take $\boldsymbol{\Sigma}$ to be the set of every $\Sigma_{t}$, so that for every $t \in I: \Sigma_{t} \in \Sigma$. These cosmic spaces $\Sigma_{t} \subset \mathcal{M}$ inherit their metric tensor $h(t)$ from $g$ on $\mathcal{M}: g=-c^{2} d t^{2}+h(t)$, where $c$ is the speed of light. Every one of the six independent components of $h(t)$, when expressed in some coordinate chart, can exhibit a different dependence on $t \in I$. Cosmic space-time structures with such erratic geometrical behavour are however rarely studied in cosmology.

In the special case of maximally symmetric cosmic space-times (having only globally isotropic and homogeneous cosmic spaces $\Sigma_{t}$ ), which have a Friedman-Robertson-Walker metric, all components of the spatial metric exhibit the same dependence on cosmic time: $h(t)=R^{2}(t) h$, where $R(t)$ is the radius of the space $\Sigma_{t}$ at cosmic time $t$. (If $R(t)$ increases and $\Sigma_{t}$ contains galaxies, then the distance between them increases because space expands over cosmic time, not because galaxies are moving away from each other through fixed space.) To these standard cosmic space-time structures Wüthrich's abysmal embarrassment argument applies. All cosmic spaces $\Sigma_{t}$ of a standard cosmic space-time have constant curvature and differ only by a scale factor. Then the Gauss curvature scalar $K_{t} \in \mathbb{R}$ of every cosmic space $\Sigma_{t}$ has the same sign. Theorem (McGabe (2004: $513)$ ): every space $\Sigma_{t}$ of curvature $K_{t}$ is isometric to either one of the following spaces $\left(\rho \equiv 1 / \sqrt{\left|K_{t}\right|}\right)$ :

[1] $K>0$ : sphere $S^{3}(\rho)$, embeddable in $\mathbb{E}^{4}$.

[2] $K=0$ : Euclidean space $\mathbb{E}^{3}$.

[3] $K<0$ : hyperboloid $H^{3}(\rho)$, embeddable in $\mathbb{E}^{3,1}$.

According to Wüthrich's formulation of the Cosmological Principle (CP), which we shall call the Universal Cosmological Principle (UCP), in cosmic space-time structures for modeling the universe, only 'cosmic' subsets of $\mathcal{M}$ represent properties of space-time points, where we define subset $P \subseteq \mathcal{M}$ to be cosmic iff per cosmic space $\Sigma_{t} \in \Sigma$, all points lie in $P$ or none of them lies $P$ :

$$
\forall t \in I: \Sigma_{t} \subseteq P \vee \Sigma_{t} \cap P=\varnothing .
$$

For further discussion about the approximate truth of CP and its role in current cosmology, we refer to Weinberg (1972: $§ 14.1 ; 2008:$ Ch. 1$)$ and McCabe (2004: 594). ${ }^{5}$

For the sake of convenience, we introduce the following sets of subsets of $\mathcal{M}$ :

\footnotetext{
${ }^{5}$ Wüthrich (2010: formula (1)) uses 2nd-order logic to formulate UCP in terms of monadic predicates, which he prefers because (i) set-theoretical representation (in 1st-order logic) is problematic when it comes to different properties having the same set-extension; and (ii) set-theory cannot deal with intensional properties (private communication by e-mail from C. Wüthrich, January 2010). When we do not identify
} 
$\operatorname{Prop}(\mathcal{M})$ : set of subsets of $\mathcal{M}$ that represent properties of points in $\mathcal{M}{ }^{6}$

$\operatorname{Cos}(\mathcal{M})$ : set of cosmic subsets of $\mathcal{M}(4)$.

$\operatorname{Aut}(\mathcal{M})$ : set of automorphic subsets of $\mathcal{M}(5)$.

(In full generality, the set-theoretical representation of a property of members of a set $\mathcal{S}$ is a subset of $\mathcal{S}$, which should not be confused with properties being identified with sets (to make the two identical). Further, if we were to take $\operatorname{Prop}(\mathcal{M})$ identical to the power-set $\mathcal{P}(\mathcal{M})$, we would make $\operatorname{Prop}(\mathcal{M})$ too encompassing, because there will be lots of subsets of $\mathcal{M}$ that do not represent any genuine properties of space-time points, let alone play a part in GTR. Mathematical entities without physical significance being there in our domain of discourse as a consequence of the mathematical background framework (set-theory) we adopt: familiar and widely accepted. Hence $\operatorname{Prop}(\mathcal{M}) \subset \mathcal{P}(\mathcal{M})$. On the other hand, when we consider every different $g$ to give rise to a different property of a space-time point, the number of different possible properties is at least that of the cardinality of the continuum $(\mathbb{R})$. Nonetheless we then still have that $\operatorname{Prop}(\mathcal{M}) \subset \mathcal{P}(\mathcal{M})$, because the cardinality of $\mathcal{P}(\mathcal{M})$ is larger than the one of $\mathbb{R}$.) Subset $P \subseteq \mathcal{M}$ is automorphic in GTR-structure $\mathfrak{G}$ iff $P$ is invariant under every automorphism:

$$
\forall \psi \in \operatorname{Aut}(\mathfrak{G}): \psi[P]=P
$$

In terms of the sets just introduced, the Universal Cosmological Principle (UCP) reads:

$$
\operatorname{Prop}(\mathcal{M}) \subseteq \operatorname{Cos}(\mathcal{M})
$$

Without a specification of $\operatorname{Prop}(\mathcal{M})$, proposition (6) cannot be decided by means of proof. One can prove that in cosmic space-times, all automorphic subsets are cosmic (see Muller $(2011)): \operatorname{Aut}(\mathcal{M}) \subseteq \operatorname{Cos}(\mathcal{M})$.

3. Space-Time Structuralism Incorrectly Characterised. Wüthrich (2010: 10451046) characterises space-time structuralism in the context of GTR as the view according to which the space-time points in $\mathcal{M}$ only exemplify intra-structural relational properties. This is fleshed out more precisely as follows.

First, structuralism should be taken to include that all and only automorphic subsets represent properties of space-time points:

$$
\operatorname{Prop}(\mathcal{M})=\operatorname{Aut}(\mathcal{M})
$$

Secondly, structuralism, as a variety of scientific realism and therefore antagonistic to bestowing objects with properties that are scientifically otiose, or worse, seem to have no

properties with their set-extensions, but keep talking in terms of sets representing properties, objection (i) evaporates; and until and unless intensional properties are presented that somehow play a rôle in GTR, objection (ii) is toothless. Furthermore, 2nd-order logic on top of set-theory surely is overdone, in dissonance with the noble spirit of parsimony. 
physical significance whatsoever (notably and notoriously haecceitas and primitive thisness), should be taken to include also Leibniz's Principle of the Identity of Indiscernibles (PIdIn) with regard to space-time points. Applied to an arbitrary GTR-structure $\mathfrak{G} \in \mathcal{G}$ (1), PIdIn says (Wüthrich (2010), formula (2), set-theoretically construed):

$$
\forall p, q \in \mathcal{M}: \operatorname{AbsInd}(p, q) \longrightarrow p=q,
$$

where absolute indiscernibility is defined as sharing all properties:

$$
\operatorname{AbsInd}(p, q, \mathcal{M}) \text { iff } \forall P \in \operatorname{Prop}(\mathcal{M}): p \in P \longleftrightarrow q \in P \text {. }
$$

We call (8) henceforth the Principle of the Identity of Absolute Indiscernibles (PIAI. So $p \in \mathcal{M}$ is by definition absolutely discernible in $\mathcal{M}$ iff it has a property that no other $q \in \mathcal{M}$ has. When we combine (7) and PIAI (8), we obtain the Principle of the Identity of Automorphic Absolute Indiscernibles (PIAAI):

$$
\forall p, q \in \mathcal{M}: \operatorname{AutAbsInd}(p, q, \mathcal{M}) \longrightarrow p=q,
$$

where $\operatorname{AutAbsInd}(p, q, \mathcal{M})$ is like $\operatorname{AbsInd}(p, q, \mathcal{M})$ save that the universal quantifier in definition $(9)$ ranges over $\operatorname{Aut}(\mathcal{M})$. PIAAI (10) is what the structuralist is committed to in addition to $(7)$.

Wüthrich (2010: 1046, our emphasis) submits that structuralism must hold that $\operatorname{Prop}(\mathcal{M})$ is "the set of automorphically invariant relations, excluding any intrinsic properties," which we take to be partly expressed by (7). Further on, it turns out that by "relations" Wüthrich means: relational properties, the sort of properties of a space-time point $p \in \mathcal{M}$ expressed by logical formulae like ' $\forall q:\langle p, q\rangle \in R$ ' and ' $\exists q:\langle q, p\rangle \in R$ ', where $R \subseteq \mathcal{M} \times \mathcal{M}$ represents some binary relation. "For the space-time structuralist described in the previous Section, $\forall F$ must range over all and only automorphically invariant properties that do not depend in their exemplification on the existence of any particular individual." (ibid., our italics).

Surely Wüthrich was moving in the correct direction when specifically mentioning relations as he did, but he failed to reach a correct destination, for relations are permitted only in so far as they give rise to extrinsic properties. So in the end Wüthrich does not take relations seriously, which is precisely what structuralism does: taking relations seriously, by acknowledging their capacity to discern (see Section 6), and not taking them only as auxiliary devices to define properties.

4. Abysmally Embarrassing Consequences. With all these preparations in position, the argument Wüthrich takes from Keränen (2001) against structuralism proceeds quickly. Let us state precisely what this argument demonstrates:

Proposition 1. Structuralism about cosmic space-times, when taken to include that all and only automorphic subsets represent properties of space-time points, and the principle 
that automorphically absolutely indiscernible space-time points are identical, is inconsistent.

The premises yield immediately that every automorphic subset $P \subseteq \mathcal{M}$ is cosmic. Then one easily proves for every $p, q \in \Sigma_{t}$ that $p \in P$ iff $q \in P$. This yields the antecedent of PIAAI (10), so that $p=q$.

The implication that $\Sigma_{t}$ contains a single point, Wüthrich (2010: 1046) also calls "the abysmal embarrassment for the space-time structuralist." We point out that Wüthrich's argument is not directed against 'primitive identity facts', also known as 'primitive thisness', for otherwise Wüthrich's argument could have been dismissed as a fallacy: the inference from indiscernibility by properties (or relations for that matter) to identity $\left(\# \Sigma_{t}=1\right)$ would then be fallacious. Proponents of 'primitive thisness' have however taken the adjective 'primitive' also as an excuse to throw off the burden of explaining what these non-qualitative identity facts are; further, an appeal to 'primitive thisness' in order to avoid Wüthrich's abysmal embarrassment is circular when not accompanied by independent argument. ${ }^{7}$ Finally, why besmirching GTR with non-qualitative properties that are physically otiose?

Let us think along Wüthrich's Keränen strategy. We first point out that a corollary of the proof of Proposition 1 is that Minkowski space-time $\mathfrak{M}(3)$ only has a single space-time point, an implication that arguably is even more if not maximally embarrassing. We can generalise above and beyond GTR to arbitrary sets.

Proposition 2. According to the Principle of the identity of Absolute Indiscernibles, every set of absolutely indiscernible objects has a single member.

The proof is trivial: if $\mathcal{S}$ is a non-empty set of whatever, and $\operatorname{Prop}(\mathcal{S})$ is the set of subsets that represent properties of whatever is in $\mathcal{S}$, and the members of $\mathcal{S}$ are absolutely indiscernible, then by PIAI (8) they are identical. We are left with a single member in $\mathcal{S}$.

For example, a line $(\mathbb{E})$ has a single point. A flat plane $\left(\mathbb{E}^{2}\right)$ has a single point. Surfaces of constant curvature, such as a sphere $S^{2}(r)$, have a single point. Euclidean space $\left(\mathbb{E}^{3}\right)$ has a single point. We can go on like this, generating embarrassments, but we won't.

Does the death bell toll for structuralism in mathematics, as Keränen (2001) argued, and for structuralism about space-time, as one could conclude on the basis of Wüthrich (2010) abysmal embarrassment argument? Or does something go deeply going wrong? We believe the last-mentioned: diagnosis and cure coming up in the next Sections.

5. Space-Time Structuralism Correctly Characterised. Section 4 has taught us that what is at stake is not only structuralism but every view that is committed to PIAI (8). Before we continue, we rehearse some terminology. Adopting the terminology of

\footnotetext{
${ }^{7}$ See Wüthrich (2010: 1049), who mentions several other authors who have discussed the possibility of 'primitive identity facts' about space-time points.
} 
Quine, as does Saunders (2003: 293), and of Muller \& Saunders (2005: 503-505), call the members of any set $\mathcal{S}$ absolutely indiscernible iff they share all their properties, and relationally indiscernible iff every member is related to all members of $\mathcal{S}$ in the same manner. Individuals are by definition absolutely discernible; the property that discerns an absolute discernible is its 'identity', its 'individuality', if you like; 'to individuate' something is to ascertain it is an individual. Members of $\mathcal{S}$ that are absolutely indiscernible but are discerned by a relation are therefore called relationals. Relationals have no identity and no individuality, they are neither absolute indiscernibles nor indiscernibles. They constitute a neglected metaphysical category. Structuralism loves relationals.

Wüthrich (2010: 1045) reports that in Max Black's canonical challenge of PIdIn, of two black spheres having a diameter of 1 mile and being 2 miles apart in further empty 3-dimensional Euclidean space $\left(\mathbb{E}^{3}\right)$, the challenge to PIdIn can be met by means of a Euclidean invariant distance relation, which discerns the spheres weakly - defined as:

$$
D(p, q) \text { iff } d_{\mathrm{E}}(p, q)>0
$$

where $d_{\mathrm{E}}: \mathbb{E}^{3} \times \mathbb{E}^{3} \rightarrow \mathbb{R}^{+}$is the Euclidean distance relation. Wüthrich (2010: 1048) actually considers this 'Exit Strategy' but levels an objection against it:

First, it will not be trivial to find such a relation defined on a homogeneous hypersurface

$\Sigma_{t}$ without disturbing its homogeneity if homogeneity is understood as defined in (1). ${ }^{8}$

Since the Euclidean space $\left(\mathbb{E}^{3}\right)$ in Black's canonical case is also homogeneous and isotropic, why would it be forbidden to use a spatial distance relation in the cosmic spaces $\Sigma_{t}$ similar to $D$ ? Relation $D(11)$ is a Euclidean invariant in that if $D(u, v)$ holds, and $\mathbf{x}(u) \in \mathbb{E}^{3}$ and $\mathbf{x}(v) \in \mathbb{E}^{3}$ are the locations of the centers of the spheres $u$ and $v$, then $D(T(u), T(v))$ also holds for every $T \in \operatorname{Sym}\left(\mathbb{E}^{3}\right)$, where $\operatorname{Sym}\left(\mathbb{E}^{3}\right)$ is the Euclidean symmetry group generated by the spatial displacements and rotations. Cosmic spaces with constant curvature have no Euclidean metric, but they do have another one, in terms of which one defines a distance function that discerns points weakly, exactly as in $\mathbb{E}^{3}$. So much for Euclidean space.

For the construction of a distance relation based on the metrical tensor $g$, we refer to Muller (2011). We shall soon, in the next Section, employ a relation based on the lightcone structure that will discern space-time points weakly. First we need to provide a correct characterisation of space-time structuralism.

Let us consider GTR-structures in all their generality. Let $\mathfrak{G}$ be a GTR-structure (1), and $p, q \in \mathcal{M}$. If $g(p) \neq g(q)$, then $g$ can be used to discern $p$ and $q$ absolutely from each other. ${ }^{9}$ But as soon as we have two points having the same $g$ and $T$, they

\footnotetext{
${ }^{8}$ Wüthrich's formula (1) is our (6), so by 'homogeneity' he understands by definition that UCP holds. This is non-standard, unless the properties are restricted to those definable in terms of $g$, because then $g$ being everywhere the same implies homogeneity.

${ }^{9}$ Identity criterion: $g(p)=g(q)$ iff $\exists \phi \in \operatorname{Diff}(\mathfrak{G}), \exists O, O^{\prime} \in \mathcal{T}(\mathcal{M}): p \in O, q \in O^{\prime}, \phi^{*} g=g$ on $O^{\prime}$, and $\phi^{\text {inv* }} g=g$ on $O$.
} 
have to be identified due to PIAI (8). This is not what we want. We want to discern non-identical points by geometrical means provided by the structure $\mathfrak{G}$, such that the discerning relation or property is automorphic. (Notice that to try to discern identical points is to try to do the logically impossible, because it is to try to refute Leibniz's Law - 'indiscernibility of identicals', which is a theorem of logic.) Of course, PIdIn should not be conceived narrowly, as requiring only absolute indiscernibility for identity, as PIAI (8) does, but requiring, more in the spirit of structuralism, in addition relational indiscernibility. In the context of GTR, the Principle of the Identity of (Absolute and Relational) Indiscernibles (PII) for space-time points reads as follows.

First we define relational indiscernibility in GTR-structure $\mathfrak{G}$ :

$$
\begin{aligned}
\operatorname{RelInd}(p, q, \mathcal{M}) \text { iff } \quad & \forall R \in \operatorname{Rel}(\mathcal{M}): \\
& ((\forall r \in \mathcal{M}:\langle p, r\rangle \in R \longleftrightarrow\langle q, r\rangle \in R) \wedge \\
& (\forall s \in \mathcal{M}:\langle s, p\rangle \in R \longleftrightarrow\langle s, q\rangle \in R)),
\end{aligned}
$$

where $\operatorname{Rel}(\mathcal{M}) \subseteq \mathcal{P}(\mathcal{M} \times \mathcal{M})$ is the set of subsets of $\mathcal{M} \times \mathcal{M}$ that represent genuine relations between space-time points. Then PII says that two space-time points are identical if they are absolutely and relationally indiscernible:

$$
(\operatorname{AbsInd}(p, q, \mathcal{M}) \wedge \operatorname{RelInd}(p, q, \mathcal{M})) \longrightarrow p=q
$$

For structuralists, who relish relations and eager to embrace PII, an additional step has to be made, in order to arrive at a genuinely structuralist PIdIn.

Analogous to definition (5) of an automorphic subset of $\mathcal{M}$, call a subset $R \subseteq \mathcal{M} \times \mathcal{M}$ to be automorphic in GTR-structure $\mathfrak{G}$ iff $P$ is invariant under all automorphisms:

$$
\forall \psi \in \operatorname{Aut}(\mathfrak{G}): \psi[R]=R
$$

Let $\operatorname{Aut}(\mathcal{M} \times \mathcal{M})$ be the set of automorphic subsets of $\mathcal{M} \times \mathcal{M}(14)$.

We now define Structuralism with respect GTR (with a capital 'S') as a view consisting of the following four principles.

0. Structural Characterisation of GTR. GTR is characterised through the set of structures of type $\mathfrak{G}(1)$.

1. Principle of Automorphic Properties and Relations. For every $\mathfrak{G}$, only automorphic subsets of $\mathcal{M}$ represent properties of space-time points and only automorphic subsets of $\mathcal{M} \times \mathcal{M}$ represent binary relations between space-time points:

$\operatorname{Prop}(\mathcal{M}) \subseteq \operatorname{Aut}(\mathcal{M})$ and $\operatorname{Rel}(\mathcal{M}) \subseteq \operatorname{Aut}(\mathcal{M} \times \mathcal{M})$

2. Principle of the Identity of Automorphic (Absolute and Relational) Indiscernibles (PII $\left.{ }^{\star}\right)$ : 
$(\operatorname{AutAbsInd}(p, q) \wedge \operatorname{AutRelInd}(p, q)) \longrightarrow p=q$

where $\operatorname{AuRelInd}(p, q)$ is like $\operatorname{RelInd}(p, q)(12)$ but replacing ' $\operatorname{Rel}(\mathcal{M})$ ' with 'Aut $(\mathcal{M} \times$ $\mathcal{M})^{\prime}$.

3. Structuralist Representation Thesis. If GTR-structure $\mathfrak{G}$ represents the universe, then every GTR-structure isomorphic to $\mathfrak{G}$ (and therefore also every structure automorphic to $\mathfrak{G})$ represents the universe.

We point out that 'possible worlds' are not mentioned in these principles. For further elaboration on these principles, see Muller (2011).

6. Discerning Space-Time Points Structurally. The next relation exploits the lightcone structure of general relativistic space-times.

Proposition 3. Space-time points in every generally-relativistic space-time are conformally and weakly discernible by a lightcone based relation; therefore absolutely indiscernible space-time points are relationals.

Proof. Consider an arbitrary GTR-structure $\mathfrak{G}(1)$. Let $L C(p)$ be the lightcone of $p \in \mathcal{M}$. Consider the following lightcone relation, that relates two points iff there is some point inside one lightcone but outside the other of these points:

$$
L(p, q) \text { iff }(\exists r \in \mathcal{M}: r \in L C(p) \backslash L C(q)) \vee(\exists t \in \mathcal{M}: t \in L C(q) \backslash L C(p)) .
$$

Then the negation of the lightcone relation $L$ is an identity criterion:

$$
\neg L(p, q) \longleftrightarrow \forall r \in \mathcal{M}(r \in L C(p) \longleftrightarrow r \in L C(q)) \longleftrightarrow p=q
$$

so that $L(p, q)$, being sufficient and necessary for $p \neq q$, discerns $p$ and $q$ weakly.

To prove that the metrical relation $L(15)$ is automorphic (14), we must prove that for every $\psi \in \operatorname{Aut}(\mathfrak{G})$, for every $p, q \in \mathcal{M}$ :

$$
L(p, q) \longleftrightarrow L(\psi(p), \psi(q))
$$

Well, proposition (17) follows from the following chain of mathematical equivalences:

$$
L(p, q) \longleftrightarrow p \neq q \longleftrightarrow \psi(p) \neq \psi(q) \longleftrightarrow L(\psi(p), \psi(q)) .
$$

The first and third 'iff' follow from the fact that $\neg L(15)$ is an identity criterion for points in $\mathcal{M}$; the second 'iff' from the fact that $\psi$ is a one-one mapping on $\mathcal{M}$. Hence we have proved something stronger than $L$ being causally invariant, namely that $L$ is permutation 
invariant because invariant under $\Pi(\mathcal{M})$, and by virtue of $(2)$ then also homeomorphically, causally, isometrically and automorphically invariant. Q.e.d.

7. Charges of Vicious Circularity. Wüthrich has however not been beaten yet, because the possibility to replace the narrow formulation of PIdIn, which is PIAI(8), with formulation PII (13), or perhaps even the structuralist formulation PII ${ }^{\star}$, which averts Wüthrich's abysmal embarrassment, was within Wüthrich's strategic purview (2010: 1048):

Secondly, in order to appeal to such relations, an individuation of objects must already be presupposed: how can I know there are at least two objects such that an irreflexive relation can be exemplified on the elements of $\Sigma_{t}$ ? I do not see how this suspicion of circularity can be dispelled.

This charge of vicious circularity is Wüthrich's final attack, aimed at exactly how we have discerned space-time points on the basis of a correct version of Structuralism. Structuralists abysmally embarrassed after all?

Nay nay. Luckily for Structuralism, we see how the suspicion of vicious circularity can be dispelled, as we shall endeavour to explain next rather succinctly (for an elaboration, see Muller (2011)).

The aim is not, when we begin with a differentiable manifold of infinitely many distinct space-time points, to find out whether there really is more than one space-time point for that would, indeed, be circular; but the aim is to find out whether the distinctness of the points can be grounded qualitatively, physically, structurally, and that has not been assumed tacitly, that can be (and now has been) demonstrated explicitly. We begin, and must begin, with distinct space-time points. The issue is whether they can be discerned qualitatively by the means provided by GTR. If they can't, then PII is in trouble and so is structuralism; if they can, PII is saved, and if they can be discerned automorphically, then also PII ${ }^{\star}$ is saved, and Structuralism stands tall. This is the issue and it has been decided by mathematical proof.

Wüthrich's final attack also fails.

8. Conclusion. In summary, Structuralism, as characterised by our four principles, does not face Wüthrich's abysmal embarrassment and is moreover able to discern spacetime points weakly by means of a physically significant relation that is invariant for the strongest relevant general symmetry transformations. This relation also discerns all spacetime from each other in the symmetric cosmic space-times which Wüthrich advanced to beat structuralism. No viscious circularities are involved, only deductive demonstrations. We therefore claim to have defeated Wüthrich's campaign against structuralism. 


\section{REFERENCES}

Earman, J. (1989). World Enough and Space-Time. Absolute versus Relational Theories about Space and Time, Cambridge, Massachusetts: MIT Press, 1989.

Keränen, J. (2001). 'The Identity Problem for Realist Structuralism', Philosophia Mathematica 9.3: $308-330$.

McCabe, G. (2004). 'The Structure and Interpretation of Cosmology. Part I: General Relativistic Cosmology, Studies in the History and Philosophy of Modern Physics 35.4 (2004) 549-595.

Muller, F.A. (2011). 'Space-Time Structuralism', in submission.

Muller, F.A., Saunders, S.W., 'Discerning Fermions', British Journal for the Philosophy of Science 59 (2008) 499-548.

Saunders, S.W. (2003). 'Physics and Leibniz's Principles', in: K. Brading and E. Castellani (eds.), Symmetries in Physics: Philosophical Reflections, Cambridge: Cambridge University Press, 289-307.

Weinberg, S. (1972). Gravitation and Cosmology: Principles and Applications of the General Theory of Relativity, New York: John Whiley \& Sons.

Weinberg, S. (2008). Cosmology, Oxford: OUP.

Wüthrich, C. (2010). 'Challenging the Spacetime Structuralist', Philosophy of Science (Proceedings PSA 2008, Part I, ed. by A. Richardson) 76.5, 1039-1051. 